Conclusions The diagnostic accuracy of the rapid 'Celiac card' test is high and similar to the sensitivity and specificity of the existing ELISA based 'Euroimmun' kit, which makes it an excellent screening test for celiac disease.

\section{IDDF2018-ABS-0009 BREAKING THE WEB: A CASE OF PLUMMER VINSON SYNDROME IN THE PHILIPPINES}

MA Marylaine Dujunco*, John Arnel Pangilinan, Joseph Bocobo. St. Luke's Medical Center, Quezon City, Philippines

\subsection{6/gutjnl-2018-IDDFabstracts.60}

Background Plummer Vinson syndrome (PVS) is an extremely rare disease characterised by the triad of iron deficiency anaemia, dysphagia and cervical esophageal web. The most accepted possible aetiology of this syndrome is iron deficiency anaemia. The prevalence of anaemia in third world countries is high and yet there is no published case to date of PVS in the Philippines.

Methods We report a case of a 44 years old female, Filipino with two decades of iron deficiency anaemia, a decade of dysphagia with a cervical esophageal web on esophagogastro-duodenoscopy (EGD).

Results After EGD with mechanical dilatation, dysphagia was resolved, and patient now is able to eat a full regular meal. She was given iron supplementation and advised annual esophagogastroduodenoscopy.

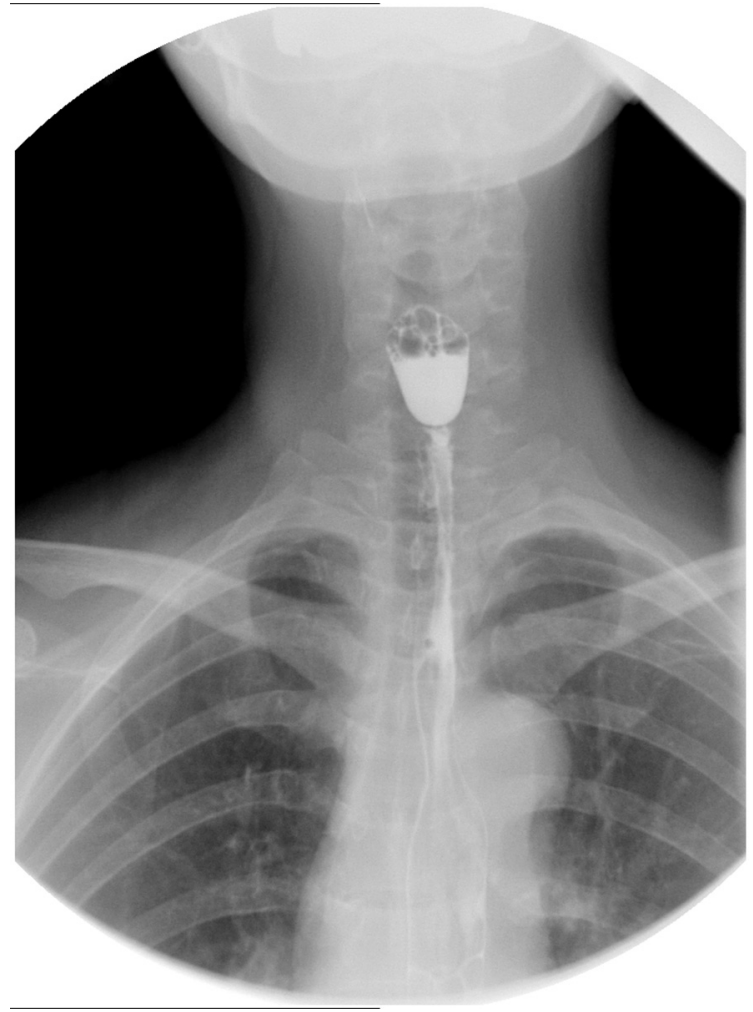

Abstract IDDF2018-ABS-0009 Figure 1

Conclusions The triad of iron deficiency anaemia, dysphagia and cervical esophageal web is Plummer Vinson syndrome which can be readily managed by iron supplementation and esophageal dilatation. Patients need to be on annual surveillance EGD due to risk for esophageal or pharyngeal malignancy.

\section{IDDF2018-ABS-0012 GALLSTONE ILEUS: A CASE REPORT ON A 30-YEAR-OLD YOUNG PATIENT AND LITERATURE REVIEW}

Winston Vequilla*. Fatima University Medical Center, Valenzuela City, Philippines

\subsection{6/gutjn|-2018-IDDFabstracts.61}

Background Gallstone ileus is a rare cause of intestinal obstruction (1\%-4\% of all cases of bowel obstruction). It affects mainly the older population with a female preponderance. A 30-year-old woman presented to the emergency room with clinical signs of intermittent intestinal obstruction. Diagnostic imaging revealed obstruction of the terminal ileum caused by a $25 \mathrm{~mm}$ calculus. The patient completely recovered after urgent surgery. This condition is very rare and more so in a young patient.

Methods A 30-year-old female presented with intermittent vomiting and abdominal pain for 7 days. She had a history of episodic epigastric pain for the last one year and a half.

Physical examination showed a moderately distended abdomen, tympanitic and hypoactive sounds. Rectal examination was normal. Pertinent ancillary tests were done with the following results:

1. Leukocytosis $(10.5 \times 109$ cells/L)

2. Plain abdominal radiograph: dilated small bowels, and air fluid levels.

Whole abdominal CT scan showed pneumobilia and adhesions in the gallbladder wall and duodenal wall, dilated small bowels and an ovoid calcific density with internal hypodensity in the small intestine lumen. The result of which is consistent with the RIGLER TRIAD seen in gallstone ileus.

Results The patient underwent immediate exploratory laparotomy. Intraoperative findings revealed an impacted gallstone measuring $10 \mathrm{~cm}$ in widest diameter located at the terminal ileum ( $40 \mathrm{~cm}$ from the ileocecal valve) causing complete bowel obstruction on the proximal part of the ileum and jejunum. The gallbladder was inspected, and a stable cholecysto-enteric fistula was noted. An enterotomy was made on the proximal non-dilated part of the ileum and revealed a large gallstone (10 $\times 4.5 \times 3 \mathrm{~cm}$ ). There was difficulty in extracting the stone, so a segmental resection of the involved ileum was done. A 2layer end-to-end anastomosis was done using silk 3.0.

The postoperative course was uneventful and was discharged improved on postoperative day 4 (hospital day 10). Conclusions In conclusion, the choice of the surgical procedure is largely determined by the clinical condition of the patient. The single-stage procedure is performed in haemodynamicallystable patients, while enterolithotomy alone is considered sufficient for unstable patients with metabolic derangements.

\section{IDDF2018-ABS-0013 THE COMBINATION OF PEMBROLIZUMAB AND BEVACIZUMAB IN THE TREATMENT OF CHOLANGIOCARCINOMA BRAIN METASTASES: CASE REPORT}

Yunqi Hua*, Xueshen Shi, Caixia Ba, Xianfeng Liu, Yongyan Yang. Department of Oncology, Baotou Cancer Hospital, Baotou, Inner Mongolia, China

\subsection{6/gutjnl-2018-IDDFabstracts.62}

Background Cholangiocarcinoma originates from primary malignant tumours of bile duct epithelial cells. Epidemiology 
shows a progressive increase in the incidence over the past decade. However, there is still no uniform treatment to the brain metastases of cholangiocarcinoma in China, especially treatment for salvage to patients with encephaledema. Here, we reported the treatment of a patient with cholangiocarcinoma brain metastases and encephaledema by using bevacizu$\mathrm{mab}$ and pembrolizumab.

Methods A 68 year old male patient, who underwent cholangiocarcinoma surgery for eight years and suffered from a headache with limited physical activity, was treated at the Chinese People's Liberation Army Navy General Hospital. The patient was in critical condition and then diagnosed as cholangiocarcinoma brain metastases with obvious cerebral oedema after MRI examination. According to the NCCN guidelines, large segmented intensity modulated radiotherapy (STRB, 84\% iso-dose curve, $8 \mathrm{~Gy} / \mathrm{f} \times 4 \mathrm{f}$ ) to the brain metastases was operated under the guidance of real-time tracking system. The patient was given bevacizumab at $200 \mathrm{mg} /$ time in combination with pembrolizumab at $100 \mathrm{mg} /$ time, once per two weeks after the peripheral blood immune function test. The therapeutic efficacy was evaluated at regular intervals.

Results MRI examination showed that the patient with obvious cerebral edema before the treatment (figure 1), then the patient's limb mobility significantly improved After three months of treatment, the cerebral edema significantly shrank, and the local metastases were obviously reduced (figure 2). After six months of continuous treatment, the MRI results showed the local metastatic lesions shrank much smaller than that three months before and maintained at a stable stage (figure 3).

Conclusions The efficiency of a combination of bevacizumab and pembrolizumab as a therapeutic treatment for cholangiocarcinoma brain metastases patient is remarkable and worthy of further clinical study. Bevacizumab has a certain function on the vascular normalisation, which can effectively enhance the clinical efficacy of immunotherapy. While reversing the surrounding immunosuppressive microenvironment of the tumour has been a new target for tumour therapy, the 'immunotherapy +molecular target therapy' for tumour therapy is of more tendency. Such combination has higher precision and lower side effects and will become the future trend for tumour treatment.

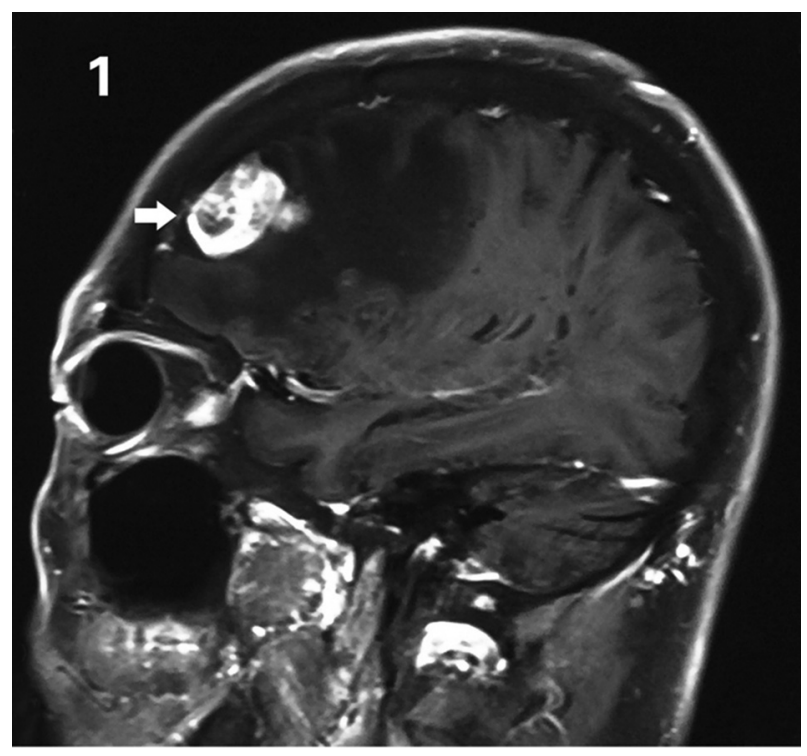

Abstract IDDF2018-ABS-0013 Figure 1

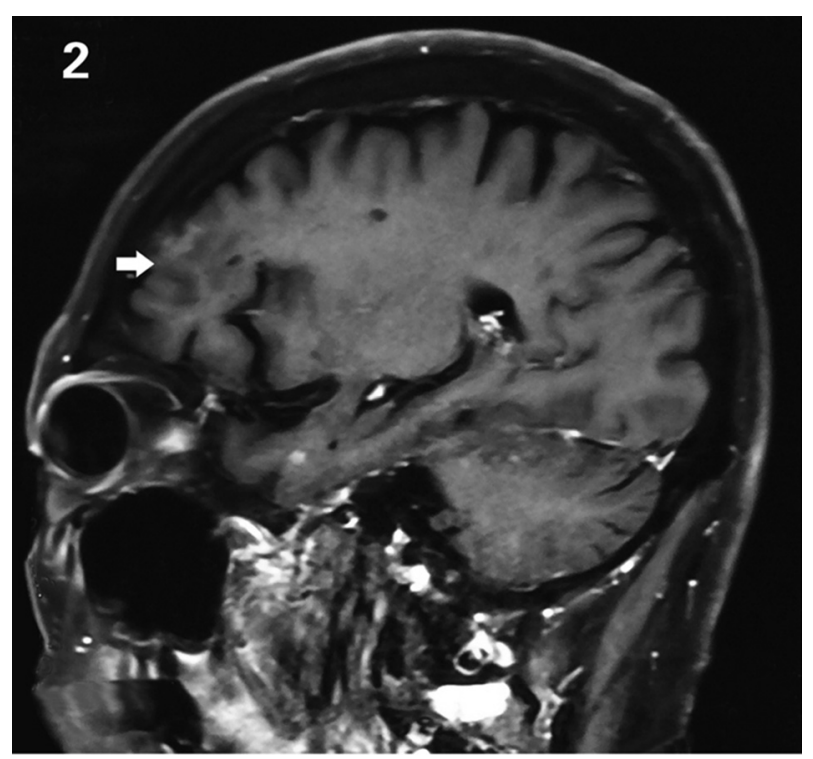

Abstract IDDF2018-ABS-0013 Figure 2

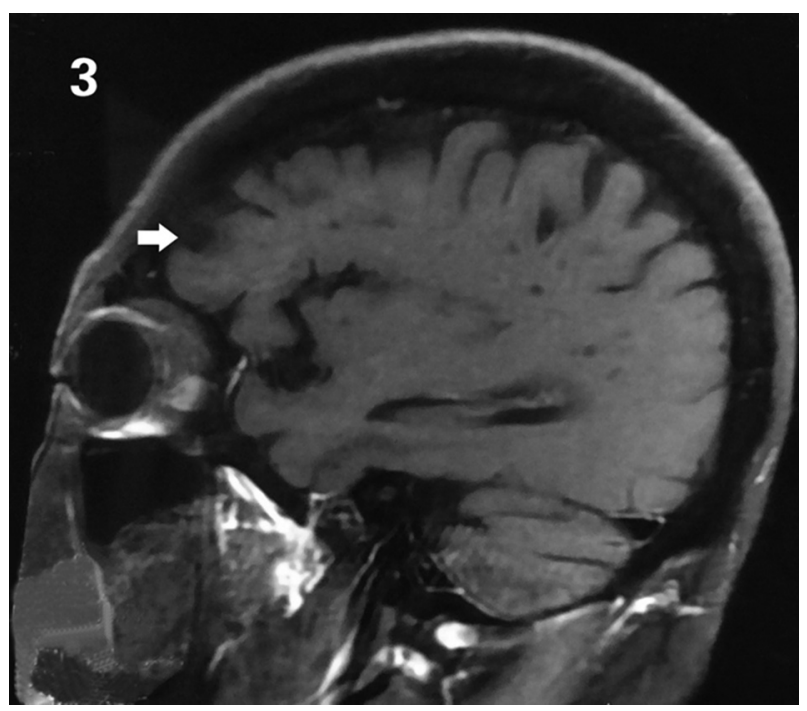

Abstract IDDF2018-ABS-0013 Figure 3

\section{IDDF2018-ABS-0014 SPYGLASS CHOLANGIOSCOPY IN BILE DUCT DISEASE: A CASE SERIES FROM NORTH INDIA}

Mukesh Nasa*, Randhir Sud. Medanta-The Medicity, India

\subsection{6/gutjnl-2018-IDDFabstracts.63}

Background Despite the use of advanced cross-sectional imaging modalities, the evaluation of bile duct disease is sometimes indeterminate and endoscopic evaluation of the bile duct is required in order to assess the final character and extent of lesions. The current study addresses the use of SpyGlass single-operator cholangioscopy in biliary strictures and biliary stones. Direct endoscopic visualisation of the biliopancreatic duct is certainly one of the greatest advances in therapeutic endoscopy. This is a case series from one of the large tertiary care institute from Northern India. 